\title{
EL ACCESO A LA EDUCACIÓN Y LA INCLUSIÓN SOCIOEDUCATIVA DE ESTUDIANTES INDÍGENAS. PROVINCIA DE SALTA-ARGENTINA
}

\author{
Nuria Macarena Rodríguez ${ }^{1}$ \\ Elisa Martina de los Ángeles Sulca ${ }^{2}$
}

Resumen: En este artículo se presenta un análisis socioeducativo de los procesos de exclusión/inclusión de los y las estudiantes indígenas que asisten al Colegio Secundario de Montañas El Alfarcito y a la Universidad Nacional de Salta, provincia de Salta, Argentina. Para ello, se toman datos obtenidos mediante entrevistas, observaciones participantes y encuestas en el marco de dos tesis de doctorado. Pese a los avances prescriptivos, el acceso a la educación -en los niveles Secundario y Universitario- en este país se desarrolla en un contexto de profundas desigualdades sociales que tiñen las experiencias escolares de estos jóvenes, lo cual da lugar a problematizar si el acceso -garantizado en las leyes- posibilita una genuina inclusión educativa.

Palabras Clave: Acceso a la educación; educación secundaria; educación universitaria; estudiantes indígenas, exclusión educativa.

\section{O acesso à educação e a inclusão socioeducativa de estudantes indígenas na Província de Salta-Argentina}

Resumo: Este artigo apresenta uma análise socioeducativa dos processos de exclusão/inclusão de estudantes indígenas que frequentam a Escola Secundária El Alfarcito de Montanhas e a Universidade Nacional de Salta, província de Salta, Argentina. Para isso, os dados obtidos por meio de entrevistas, observações dos participantes e pesquisas são coletados no âmbito de duas teses de doutorado. Apesar dos avanços prescritivos, o acesso à educação - nos níveis secundário e superior - neste país é desenvolvido em um contexto de profundas desigualdades sociais que mancham as experiências escolares desses jovens, o que abre espaço para problematizar se o acesso - garantido nas leis - permite uma inclusão educacional genuína.

Palavras-chave: Acesso à educação; ensino médio; ensino universitário; estudantes indígenas; exclusão educacional.

\footnotetext{
${ }^{1}$ Universidad Nacional de Tucumán/Argentina (nuria.macarena.rodriguez@gmail.com)

${ }^{2}$ Universidad Nacional de Salta/Argentina (elysulca@gmail.com)
}

$$
\begin{array}{lll}
\text { v. } 22 \text { n. } 51 \quad \text { jul/set } 2020
\end{array}
$$




\section{Access to education and socio-educational inclusion of indigenous students in the Province of Salta-argentina}

Abstract: This article presents a socio-educational analysis of the processes of the exclusion/inclusion of the male and the female indigenous students who asist to the High School of Montains "El Alfarcito" and to the National Univesity of Salta, Province of Salta, Argentine. For it, obteined data is taken from interviews, participant observations and surveys, whitin the framework of two doctoral theses. Dispite the prescriptive progress, the access to education in this country - on secondary and university levels -, it developes in a context of deep social inequalities, that they dye the school experiencies of these young people, which gives rise to problematize if the access - guaranted in the laws - makes posible a genuine educational inclusión.

Keywords: Education Access; Secondary education; University education; indigenous students; Educative exclusion.

\section{INTRODUCCIÓN}

El extenso territorio argentino es habitado por grupos sociales diversos, cuyas realidades educativas son desiguales. La organización política federal del país posibilita que cada jurisdicción tenga competencias en materia educativa, siendo el nivel Superior Universitario el único que continúa gestionado por el Estado Nacional, aunque las universidades públicas gozan de autonomía en su gobierno. Por lo expuesto, no es posible hablar de las relaciones entre Estado y Comunidades Indígenas en términos hegemónicos en las diferentes provincias de Argentina. En este sentido un análisis de las políticas educativas en materia indígena cobrará diferentes significados según el contexto jurisdiccional ${ }^{3}$ en el que se enfoque.

El objetivo de este artículo es analizar dos casos de experiencias con la diversidad cultural en educación: por un lado, el Colegio Secundario de Montañas El Alfarcito, institución pública de gestión privada al que asisten jóvenes que se autorreconocen al pueblo indígena Tastil. Por otro lado, la Universidad Nacional de Salta, única Universidad Pública de la provincia, que cuenta con una sede central -en la ciudad de Salta- y tres sedes regionales en el

Actualmente, Argentina está dividida en 24 jurisdicciones: 23 provincias y la Ciudad Autónoma de Buenos Aires. 
interior de la provincia, en la cual desde el año 2010 se implementó un "Proyecto de tutorías con los Estudiantes de Pueblos Originarios".

A partir de los datos obtenidos mediante entrevistas, observaciones participantes y encuestas en el marco de dos tesis de doctorado se analiza los procesos de exclusión/inclusión de los y las estudiantes implicados en los casos mencionados. El fin de dicho análisis es establecer diferencias y puntos en común que permitan evidenciar las condiciones reales de acceso y permanencia de estos jóvenes y las posibilidades de traducirse -o no- en inclusión educativa.

A pesar de los innegables avances en el acceso a la educación de los sectores históricamente excluidos, en los niveles secundario y universitario este acceso coexiste con significativas brechas socioeconómicas y culturales, que atraviesan las trayectorias educativas de los y las estudiantes indígenas. A esto se le suma el carácter netamente moderno y occidentalizado de las instituciones de estos niveles y la carencia de un abordaje real desde una perspectiva intercultural.

En las siguientes páginas se presentan algunas precisiones teóricas en torno a ciertos conceptos considerados importantes para la compresión del posterior desarrollo del artículo, así como un abordaje descriptivo de los marcos normativos que tienen incidencia en las instituciones de educación secundaria y universitaria y en el abordaje de la diversidad cultural, cimientos para el posterior análisis. Luego se precisarán aspectos metodológicos referentes a las instancias de recolección de datos y análisis de información. Seguidamente, se desarrolla el análisis a partir de la información relevada. Para cerrar se esbozan conclusiones en torno a la problemática abordada y se plantean nuevos interrogantes para un trabajo futuro.

\section{LINEAMIENTOS TEÓRICOS}

Para el análisis socioeducativo son necesarias algunas aproximaciones teóricas que permitan entender el abordaje del trabajo. En este sentido, es preciso entender que las categorías teóricas no son ingenuas en tanto comprenden significados diversos que consideramos importante explicitar. 


\section{Acerca de los conceptos de inclusión/exclusión}

La inclusión como principio en las normativas internacionales y nacionales se estableció como regla excepcional. El reconocimiento de los diversos grupos sociales -pueblos indígenas, personas con discapacidad, entre otros- derivó en una tendencia declarativa de la inclusión. Los grupos históricamente excluidos ahora deben ser parte del sistema social, político y educativo. Así pues, el carácter que tiene la inclusión es enunciativo, teórico y prescriptivo, aunque ha generado paulatinos cambios y avances en el acceso a derechos de los pueblos indígenas. En este aspecto, cabe la pregunta ¿cuál ha sido la participación de los pueblos en la elaboración de las normativas que los comprende? ¿inclusión a qué? ¿de qué manera?

En este trabajo se analiza la coexistencia de los procesos de inclusión y exclusión por lo que es necesario precisar qué implica cada uno de ellos para luego entender su conjunción en el ámbito de las dinámicas institucionales.

La inclusión comprende intervenciones y acciones que tienden a garantizar el derecho a acceder y permanecer en el sistema educativo de los diversos grupos sociales. En los últimos años la atención a la diversidad se traduce como la inclusión de estos sectores al sistema económico, político y educativo (SINISI, 2010). De allí que la inclusión se establece como meta, como objetivo a ser cumplido por las instituciones sociales y escolares.

Por su parte, la exclusión social imposibilita el acceso a una serie de recursos y actividades económicas, sociales, políticas y culturales a personas y grupos vulnerables. La exclusión niega el acceso a determinados derechos vitales, tales como trabajo, vivienda, salud, educación, tiempo libre, entre otros. Asimismo, la exclusión social deriva, en cierto modo, en una exclusión escolar. Dubet (2005) manifiesta que las diversas exclusiones sociales no son más que medidas externas que afectan a la escuela y se reproducen en su interior.

Resulta importante aclarar que no existe una relación proporcional entre exclusión e inclusión, sino que son procesos interrelacionados que ocurren simultáneamente. Mayores acciones de inclusión no disminuirán proporcionalmente las formas de exclusión e incluso pueden generar otras manifestaciones de ésta (WIGDOROVITZ DE CAMILLONI, 2008). De hecho, pensar en que un grupo plantea acciones de inclusión para otro, representa un modo de exclusión, en tanto fortalece ciertas relaciones de dominación. Por ello es importante reconocer que "la exclusión es una relación 
social y no un estado o posición ocupada en la estructura institucional de una determinada sociedad" (GENTILI, 2009, p. 34).

Específicamente, cuando se hace referencia a la inclusión de estudiantes indígenas, el señalamiento de la diferencia cultural en los espacios formativos debe estar atento a no convertir las diferencias estructurales en estigmas. Para abordar estos fenómenos en el sistema educativo es preciso poner de relieve lo que establecen las normativas vigentes que apelan a los derechos vitales de los pueblos indígenas y los modos en que se efectivizan o no en las instituciones y sus prácticas.

\section{Marcos legales nacionales e internacionales}

Argentina es un país habitado por la diversidad cultural y lingüística, el último Censo poblacional llevado adelante por el Instituto Nacional de Estadísticas y Censos (INDEC, 2010) registra que 955.032 personas se autoperciben indígenas o descendientes de algún pueblo indígena u originario, sobre un total de 40.117.096 de habitantes. En la provincia de Salta 79.204 personas se autorreconoce como indígenas o descendientes de algún pueblo indígena u originario, sobre el total de 1.214.441 habitantes, lo que representa un porcentaje del $6,5 \%$, esto es cercano al triple de la cifra nacional de $2,4 \%$ (INDEC, 2010). Además, se ubica en el quinto lugar en relación a provincias con mayor población indígena.

Es importante destacar que Salta es la provincia que alberga la mayor diversidad de pueblos indígenas. La encuesta censal detalló 31 pueblos indígenas en el total País: Atacama, Ava Guaraní, Aymara, Chané, Charrúa, Chorote, Chulupi, Comechingón, Diaguita-Calchaquí, Guaraní, Huarpe, Kolla, Lule, Maimará, Mapuche, Mbyá Guaraní, Mocoví, Omaguaca, Ona o Selk'man, Pampa, Pilagá, Quechua, Rankulche, Sanavirón, Tapiete, Tehuelche, Toba o Qom, Tonocote, Tupí Guaraní, Vilela, Wichí. Sin embargo, el ENOTPO (Encuentro Nacional de Organizaciones Territoriales de Pueblos Originarios) registra la existencia de otros pueblos no consignados por el INDEC -ya sea por su reciente reconocimiento o por la carencia de personería jurídica al momento de ser censados- tales como: Chicha, Guaycurú, Ocloya, Avipon, Yamanas o Yaganes, Querandi, Tastil, Tilian, Tilcara, Iogys, Nivaclé, Weenhayek. Probablemente estos pueblos se encuentren registradas en la categoría "otros" de las encuestas censales. El criterio utilizado en este relevamiento para la identificación de las personas indígenas y su pertenencia a 
los distintos pueblos fue el autorreconocimiento. El mapa $\mathrm{N}^{\circ} 1$ representa la diversidad de pueblos indígenas en el país, diferenciados por provincias, cabe aclarar que no refleja datos sobre cantidad de habitantes ni ubicaciones específicas al interior de cada jurisdicción.

Figura 1: Diversidad de Pueblos Indígenas por provincias en Argentina.

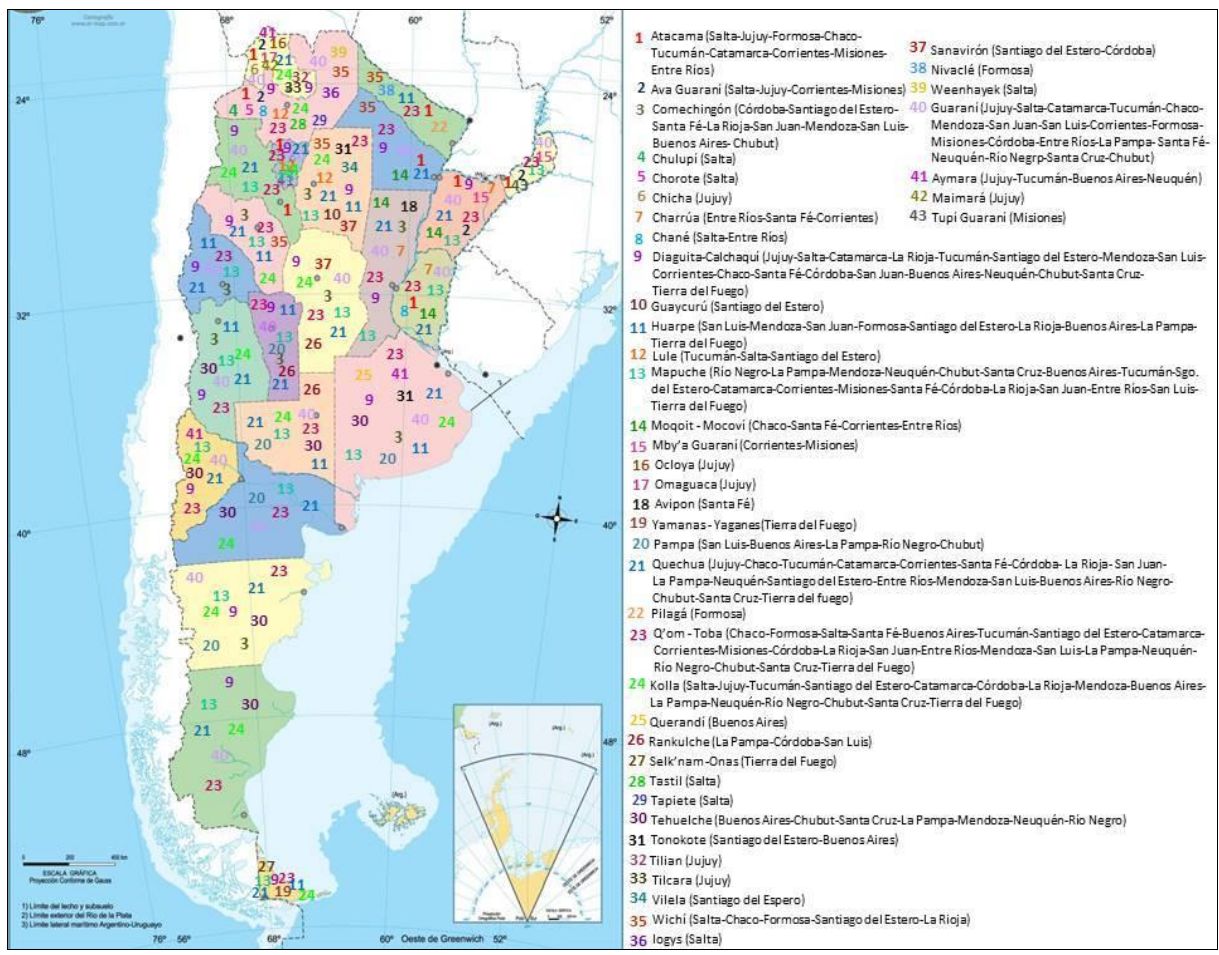

Fuente: Elaboración en base a datos del Censo 2010 (INDEC) y ENOTPO (Encuentro Nacional de Organizaciones Territoriales de Pueblos Originarios, 2015)

Según los datos del Censo (INDEC, 2010), en Salta se encuentran ocho pueblos indígenas: Wichí, Kolla, Guaraní, Ava Guaraní, Diaguita-Calchaquí, Toba, Chané y Chorote. Según estos datos, la mencionada provincia no tendría la mayor diversidad de pueblos, dado que otras provincias registran hasta nueve. Sin embargo, si se considera a los pueblos que no fueron censados por este organismo, realizando el cruce con la información más actualizada que proporciona ENOTPO (2015), Salta resulta ser la más diversa con la presencia 
de quince pueblos indígenas en su territorio. En el mapa $\mathrm{N}^{\circ} 2$ se representa la diversidad de pueblos indígenas en la provincia, con una estimación de la ubicación en relación a las características geográficas en las que habitan las comunidades, cabe aclarar que no refleja datos sobre cantidad de habitantes, dado que no existe un relevamiento fehaciente al respecto en relación a los pueblos no registrados por el Censo.

\section{Figura 2: Diversidad de Pueblos Indígenas en la Provincia de Salta y ubicación geográfica estimativa.}

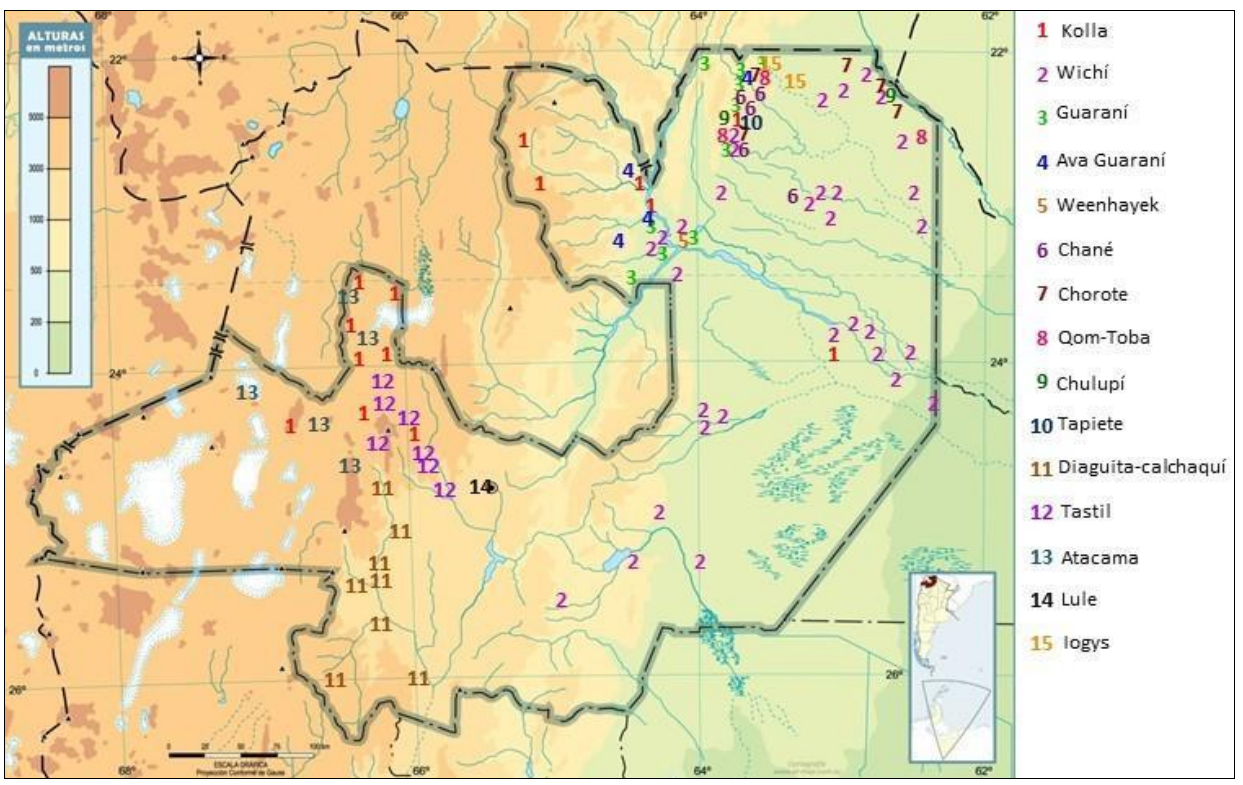

Fuente: Elaboración en base a datos del Censo 2010 (INDEC), ENOTPO (Encuentro Nacional de Organizaciones Territoriales de Pueblos Originarios, 2015) e INAI (Instituto Nacional de Asuntos Indígenas, 2019)

El relevamiento, realizado mediante el Censo Nacional, respecto a la población indígena que habita el país se inscribe en marcos normativos internacionales y nacionales. Es decir, se constituye en un intento por dar cumplimiento a los convenios internacionales y a la Constitución Nacional mediante el cual se responde -también- a una deuda histórica con estos pueblos. 
En la tabla $\mathrm{N}^{\circ} 1$ se presentan las normativas indigenistas desde 1989, momento en el que se firma el Convenio 169 de la Organización Internacional del Trabajo (OIT). Este instrumento internacional establece los derechos colectivos de los pueblos indígenas, destacando los siguientes: acceso a la tierra, al trabajo, a la salud y a la educación. Asimismo, el convenio destaca el autorreconocimiento como criterio fundamental para identificar a estos grupos.

Tabla 1: Normativas internacionales, nacionales y provinciales sobre los Pueblos Indígenas

\begin{tabular}{|l|l|l|l|}
\hline Año & $\begin{array}{l}\text { Normativa } \\
\text { Internacional }\end{array}$ & $\begin{array}{l}\text { Normativa Nacional } \\
\text { Convenio 169 de la } \\
\text { Organización } \\
\text { Internacional del } \\
\text { Trabajo sobre Pueblos } \\
\text { Indígenas y tribales. }\end{array}$ & $\begin{array}{l}\text { Normativa de la } \\
\text { Provincia de Salta }\end{array}$ \\
\hline 1992 & & $\begin{array}{l}\text { Ley 24.071 } \\
\text { "Apruébase el } \\
\text { Convenio 169 de la } \\
\text { Organización } \\
\text { Internacional del } \\
\text { Trabajo sobre Pueblos } \\
\text { Indígenas y Tribales en } \\
\text { Países Independientes" }\end{array}$ & \\
\hline 1993 & & $\begin{array}{l}\text { Ley No 24.195 “Ley } \\
\text { Federal de Educación” }\end{array}$ & \\
\hline 1994 & & $\begin{array}{l}\text { Reforma } \\
\text { Constitucional de la } \\
\text { República Argentina }\end{array}$ & \\
\hline & & & $\begin{array}{l}\text { Ronstitución provincial } \\
\text { salteña. }\end{array}$ \\
\hline
\end{tabular}




\begin{tabular}{|c|c|c|c|}
\hline Año & $\begin{array}{l}\text { Normativa } \\
\text { Internacional }\end{array}$ & Normativa Nacional & $\begin{array}{l}\text { Normativa de la } \\
\text { Provincia de Salta }\end{array}$ \\
\hline 2000 & & $\begin{array}{l}\text { Ratificación por el } \\
\text { Poder Ejecutivo del } \\
\text { Convenio } 169 \text { de la } \\
\text { Organización } \\
\text { Internacional del } \\
\text { Trabajo }\end{array}$ & $\begin{array}{l}\text { Ley } 7.121 \text { de } \\
\text { desarrollo de los } \\
\text { Pueblos Indígenas }\end{array}$ \\
\hline 2001 & $\begin{array}{l}\text { Declaración Universal } \\
\text { de la UNESCO sobre } \\
\text { la diversidad cultural }\end{array}$ & & \\
\hline 2006 & & $\begin{array}{l}\text { Ley } 26.160 \text { de } \\
\text { emergencia en la } \\
\text { propiedad comunitaria } \\
\text { indígena, con tres } \\
\text { prórrogas }(2009,2013, \\
2017) \\
\text { Ley } 26.206 \text { "Ley de } \\
\text { Educación Nacional" }\end{array}$ & \\
\hline 2007 & $\begin{array}{l}\text { Declaración de las } \\
\text { Naciones Unidas sobre } \\
\text { los derechos de los } \\
\text { pueblos Indígenas. }\end{array}$ & & \\
\hline 2008 & & & $\begin{array}{l}\text { Ley } 7.546 \text { "Educación } \\
\text { de la Provincia de } \\
\text { Salta" }\end{array}$ \\
\hline
\end{tabular}

Fuente: Elaboración propia.

Respecto a la educación, esta normativa plantea:

- El deber de garantizar -en igualdad al resto de la población nacional- la adquisición de una educación en todos los niveles Art. 26. 
- $\quad$ El deber de enseñar a leer y escribir en la propia lengua - Art. 28.

- El deber de propiciar el acceso a conocimientos y aptitudes que permitan participar en la propia comunidad y en la comunidad nacional - Art. 29.

En Argentina, este Convenio se ratificó por el Poder Legislativo en el año 1992 y por el Poder Ejecutivo en el 2000, entrando en vigencia a partir del año 2001.

En el año 1993 se sanciona la Ley Federal de Educación № 24.195 (LFE) que tiene vigencia hasta el 2006 -inclusive-, año en el que se aprueba la Ley de Educación Nacional №26.206 (LEN), que entra en vigencia en el 2007. En la LFE se establece la "garantía" de una educación básica común para todos los niños y adolescentes del país -Art. 15 Inc. A-, además se hace referencia a iniciar acciones para el "rescate y fortalecimiento de lenguas y culturas indígenas, enfatizando su carácter de instrumento de integración” -Art 34-. Por su parte, la LEN extiende la obligatoriedad de la educación hasta el nivel secundario y asegura que su cumplimiento se viabilizará mediante instituciones que se ajusten a los requerimientos locales y comunitarios -Art. 16-. Además, esta Ley dispone el respeto a la lengua y la identidad cultural de los pueblos indígenas mediante la promoción de la "multiculturalidad" en la educación de todos y todas -Art. 11, Inc. $\mathrm{N}$ - y establece que formará parte de los contenidos curriculares comunes a todas las jurisdicciones el conocimiento sobre la diversidad cultural de los pueblos indígenas y sus derechos -Art. 92 Inc. E-.

Por otro lado, en su capítulo XI "Educación Intercultural Bilingüe" -Art. 52, 53 y 54- la LEN establece la Educación Intercultural Bilingüe (EIB) como la modalidad de educación que garantizará el cumplimiento del Art. 75 Inc. 17 de la Constitución Nacional. La reforma constitucional del 1994, en adhesión al Convenio 169 de la OIT, reconoce -en mencionado inciso- la preexistencia étnica y cultural de los pueblos indígenas, "garantiza" el respeto a su identidad y el derecho a una educación intercultural bilingüe, a su vez que reconoce las personerías jurídicas de sus comunidades y la posesión comunitaria de las tierras que ocupan.

Respecto a la EIB, la LEN -Art. 53- establece que el Estado se hará responsable de: 
- Crear mecanismos para que los y las representantes de los pueblos indígenas tengan participación permanente en los órganos responsables de definir y evaluar las estrategias de EIB.

- Garantizar la formación docente.

- Impulsar la investigación en temática indígena que pueda servir de insumo para la elaboración de propuestas pedagógicas y materiales educativos.

- Promover instancias institucionales de participación de los pueblos indígenas en la planificación y gestión de la EIB

- Propiciar la construcción de modelos y prácticas educativas propias de los pueblos indígenas que incluyan sus rasgos culturales.

Retomando las normativas internacionales, se encuentra la Declaración Universal sobre la Diversidad Cultural de la UNESCO (2001), la cual manifiesta que la diversidad cultural comprende un patrimonio de la humanidad que debe ser reconocida y consolidada en beneficio de las generaciones presentes y futuras -Art 5-. Asimismo, establece que la defensa de la diversidad cultural debe ser un imperativo ético, inseparable del respeto a la dignidad humana. Lo que supone el compromiso de respetar los derechos humanos y las libertades fundamentales, en particular los derechos de las personas que pertenecen a minorías y los de los pueblos autóctonos.

Respecto a la educación, este documento sostiene que toda persona tiene derecho a: una educación de calidad que respete plenamente su identidad cultural, la posibilidad de expresarse, crear y difundir sus obras en la lengua que desee y en particular en su lengua materna, la posibilidad de participar en la vida cultural que elija y conformarse a las prácticas de su propia cultura.

Por su parte, la Declaración de las Naciones Unidas sobre los derechos de los pueblos indígenas establece que los indígenas, como pueblos o como personas, deben disfrutar plenamente de todos los derechos humanos y las libertades fundamentales -Art. 1-. En este sentido -y específicamente en lo atinente a educación-, este instrumento internacional -Art. 14- reconoce el derecho de los pueblos a: establecer y controlar sus sistemas e instituciones 
docentes, en sus idiomas y conforme a sus métodos culturales de enseñanza y aprendizaje; acceder a todos los niveles y formas de educación del Estado, sin discriminación; que el Estado -junto con los pueblos indígenas- adopte medidas en relación a posibilitar que las personas indígenas -principalmente niños y niñas- tengan acceso a educación en su propia cultura e idioma.

Por otro lado, en 2006 se sanciona en Argentina la Ley de Emergencia Territorial indígena $\mathrm{N}^{\circ} 26.160$ que declara la emergencia en materia de posesión y propiedad de la tierra que ocupan los pueblos indígenas. El principal objetivo fue realizar un relevamiento técnico-jurídico-catastral de las comunidades indígenas registradas por el Instituto Nacional de Asuntos Indígenas (INAI) o por los organismos jurisdiccionales competentes. Ante el incumplimiento de los objetivos de esta Ley, se establecieron dos prórrogas en el año 2009 y 2017.

En cuanto a normativa provincial, en Salta se realiza la reforma constitucional en 1998, en la cual se reconocen los mismos derechos y en el mismo sentido que en la Constitución Nacional del 1994. Luego, en el año 2000, se sanciona en la provincia la Ley 7.121 de desarrollo de los Pueblos Indígenas, en base a lo establecido en ambas constituciones. En el marco de esta Ley se crea el Instituto Provincial de los Pueblos Indígenas de Salta (IPPIS), institución autárquica, dependiente del poder ejecutivo y que se hará responsable de promover acciones en distintas áreas como Salud, Tierras, Economía, etc. En lo que respecta a la educación, esta ley adjudica al IPPIS la promoción de acciones para el logro de los siguientes objetivos:

- Propiciar un Sistema Educativo que beneficie a los pueblos indígenas, en base a un vínculo de intercambio cultural y por medio de la formulación de bases curriculares adecuadas a cada contexto.

- Regionalizar la enseñanza tomando como referencia la cultura de los educandos para que gradualmente se acerquen al contexto cultural global.

- Promover la formación de docentes especializados en educación indígena

- Gestionar con quienes corresponda la ejecución de campañas intensivas de educación de adultos para reducir el analfabetismo y promover la educación bilingüe. 
- Facilitar el pase de los alumnos entre establecimientos educativos, para evitar la repitencia y deserción como consecuencia de la característica cultural de semi-nomadismo de algunos pueblos.

- Posibilitar el acceso de educandos de cada grupo étnico a carreras docentes de nivel secundario, terciario y universitario, por medio de un sistema de becas.

Finalmente, la Ley de Educación de la Provincia $\mathrm{N}^{\circ} 7.546$-Art 8 Inc. Westablece como uno de los principios, fines y criterios de la educación en Salta "asegurar a los pueblos indígenas el respeto a su lengua y su identidad cultural". El capítulo VII es el específico sobre EIB y no presenta diferencia alguna respecto al capítulo XI de la LEN.

Este recorrido por las normativas internacionales, nacionales $\mathrm{y}$ provinciales permite evidenciar un avance en el reconocimiento de derechos, el cual se enmarca en la reorganización y lucha persistente por parte de los distintos pueblos indígenas que habitan el país. Es decir, el acceso a derechos fundamentales no es mérito del Estado, sino de los movimientos indígenas. Además, resulta importante destacar que existe una distancia evidente entre lo que prescriben estas normativas y los efectos prácticos; entre lo establecido en las normas jurídicas y las prácticas institucionales concretas (MATO, 2015).

\section{ASPECTOS METODOLÓGICOS}

El abordaje metodológico de la investigación es fundamentalmente cualitativo. No obstante, se contemplaron aproximaciones cuantitativas de carácter descriptivo a fin de relevar datos que posibiliten un abordaje complejo del fenómeno estudiado.

\section{Poblaciones en estudio}

Las poblaciones que forman parte de este trabajo son: los estudiantes de la comunidad de Las Cuevas -pueblo indígena Tastil- que asisten al Colegio Secundario de Montañas El Alfarcito y los estudiantes indígenas que asisten a la Universidad Nacional de Salta (UNSa) y participan del Proyecto de Tutorías con los Estudiantes de Pueblos Originarios (ProTconPO) en dicha institución. 


\section{Técnicas de recolección de datos}

Las técnicas empleadas para la recolección de datos fueron entrevistas en profundidad, observaciones participantes y encuestas. Los cuestionarios se aplicaron a todos los y las estudiantes de la Comunidad de las Cuevas, Pueblo Tastil, tuvieron como finalidad relevar datos que permitieron un acercamiento general más que en profundidad de la población en estudio.

Con las entrevistas en profundidad y la observación participante se buscó relevar datos que permitieron conocer, interpretar y comprender la producción de sentidos por parte de los y las jóvenes estudiantes en relación a la escuela secundaria y la Universidad a la que asisten y lo que significa para ellos acceder a estos niveles de educación. Las observaciones participantes se realizaron en diferentes actividades realizadas en ambas instituciones.

En cuanto a la selección de los sujetos a entrevistar, y en correlación con la lógica cualitativa del estudio, se realizó un muestreo intencional mediante el cual se seleccionan los casos por su capacidad para generar información relevante (YUNI y URBANO, 2016). En los estudios cualitativos, la representatividad de la muestra está vinculada más a la significatividad que a la cantidad de casos, en tanto cada uno de los informantes presenta particularidades a partir de las cuales se realiza el análisis, así pues, las muestras tienden a ser intensivas: se estudian pocos casos, pero en mayor profundidad (YUNI y URBANO, 2016).

En base a lo planteado, se seleccionaron, para el caso de la Universidad Nacional de Salta, cinco participantes, teniendo como criterio que presenten situaciones diferentes respecto a las tutorías: Por un lado, la autora del proyecto y coordinadora de los espacios de tutorías. Por otro, dos tutoras, una perteneciente a una comunidad indígena, que aún no finalizó la carrera y que ha perdido contacto con las tutorías; y una no-indígena, que se graduó y aún colabora con las tutorías. Y, finalmente, dos tutelados que participan en la mayor parte de las actividades propuestas desde el espacio de tutorías -en este punto es importante aclarar que las tutorías con pueblos originarios son de participación voluntaria-. Para el caso de la escuela secundaria se seleccionaron al total de estudiantes que se autorreconocen indígenas (dieciséis) ya que a la misma también asisten estudiantes que se autorreconocen criollos. 


\section{ANÁLISIS SOCIOEDUCATIVO}

En las últimas décadas la ampliación de la obligatoriedad en el sistema educativo argentino implicó la incorporación de jóvenes de sectores históricamente excluidos. En este marco, la obligatoriedad de la educación secundaria, de carácter históricamente selectivo, ha impactado en las expectativas sociales y educativas de los y las jóvenes indígenas, así como en sus trayectorias de vida, permitiendo a muchos de ellos constituirse en la primera generación familiar en acceder a este nivel y en algunos casos a la Universidad. La información recabada da cuenta de ello: los y las estudiantes secundarios y universitarios encuestados y entrevistados aseguran ser -ellos o sus hermanos coetáneos- los primeros en acceder a tales niveles en su historia familiar.

Lo referido anteriormente no es un dato anecdótico, sino que reviste importancia al momento de analizar las condiciones materiales y simbólicas de acceso a estos niveles, desde las cuales los estudiantes van configurando sentidos en torno a las instituciones educativas y sus horizontes a futuro.

Siguiendo a Bourdieu (1998) el acceso a la educación de los y las estudiantes indígenas puede ser analizado desde la noción de capital cultural para dar cuenta de los procesos de apropiación y significación del capital escolar que ofrecen las instituciones educativas. En este sentido -al igual que el capital económico- el capital cultural se distribuye de forma desigual, es decir no todos los y las estudiantes tienen las mismas oportunidades de acceder y apropiarse de los conocimientos escolares. La hipótesis del capital cultural de Bourdieu busca explicar los mecanismos de desigualdad que rigen al interior de las instituciones y que van configurando subjetividades, identidades. Es innegable que las instituciones educativas -principalmente la Universidad, pero también los otros niveles- continúan reproduciendo la cultura occidental, dominante, como universal. Así pues, bajo esas reglas de juego, la noción de capital cultural de Bourdieu (1998) se ajusta a la lógica imperante en el sistema educativo argentino.

Resulta evidente la situación de desventaja en la que acceden los estudiantes indígenas a las instituciones educativas -que en Argentina distan mucho de ser interculturales-. En este contexto de desigualdades persistentes, demandas y posibilidades: ¿es posible hablar de una inclusión genuina de los jóvenes indígenas al sistema educativo? La garantía de acceso ¿ ¿se traduce en inclusión socioeducativa? Sin la intención de dar respuestas acabadas a estos 
interrogantes, se intenta problematizarlos a partir de la información relevada y de los marcos teóricos de referencia.

\section{El acceso a la educación en relación a la inclusión socioeducativa de jóvenes indígenas}

Si bien el acceso a la educación se enmarca en distintas normativas nacionales e internacionales- la garantía de ese derecho se quebranta en los espacios escolares concretos al no generar transformaciones estructurales que incluyan los conocimientos, las prácticas culturales y cosmovisión de los pueblos indígenas. La incorporación a las estructuras organizativas del sistema educativo acaba por debilitar, ocultar y muchas veces desmoronar las identidades indígenas de los y las jóvenes, en tanto la cultura y organización escolar, las prácticas docentes, los contenidos a enseñar van en detrimento de la propia identidad y cultura.

En este sentido, el acceso a la educación tiene lugar en la dinámica inclusión- exclusión. Así pues, en el intento por "incluir a todos”, la escuela puede "actuar acallando lo diferente, excluyendo identidades que, en lugar de ser reconocidas en su valor propio, en sus formas particulares de expresión, terminan siendo compulsivamente obligadas a mimetizarse con la finalidad homogeneizante que signó el sistema educativo en sus orígenes y que consiste básicamente en la imposición de una cultura única y el exterminio literal de otras formas culturales" (NARODOWSKI, 2008, p.21).

En relación a lo enunciado, una tutora de pueblos originarios relata experiencias -de los estudiantes indígenas que participan en el proyecto- que permiten evidenciar estas tensiones entre procesos de inclusión-exclusión en el acceso a la educación en la Universidad:

Los textos te hablan de conceptos que en su lengua materna no existen. Entonces, es tratar de encontrar y de contextualizar esa palabra. Eh... es un trabajo muy abstracto y es un triple o cuatro veces mayor el esfuerzo que tenés que hacer para comprender un texto, para leer un texto, leer una página de un texto. Entonces es un trabajo muy individual, agotador diría yo, cansador y hasta frustrante para el estudiante (...) yo te digo hasta frustrante, porque esto el hecho de que... de llegar a decir "la pucha yo soy el que no entiendo" es como mi culpa, es como que es mi problema (Entrevista a colaboradora y ex-tutora del ProTconPO, pág. 4 lín. 129-138). 
El fragmento resulta significativo si se contempla que el idioma es un rasgo cultural importante en muchos pueblos indígenas y que debe ser dejado de lado en instituciones educativas como las que se analizan en este trabajo. Los estudiantes a quienes refiere esta tutora accedieron a la educación, pero siguen siendo excluidos en los términos planteados por Narodowski (2008). Además, la cuestión del lenguaje no es accesoria, el hecho de que una palabra no exista en determinado idioma puede evidenciar que para esa cultura no fue necesario referir a ese concepto. Así pues, el fragmento plasma claramente la imposición cultural que se interpreta como incompatible con la idea de inclusión a la que se referencia cuando se habla de pueblos indígenas.

Otra cuestión que interesa analizar del discurso es la autorresponsbilización de las dificultades en la apropiación del conocimiento. Kaplan (2008) sostiene que las representaciones que se esgrimen acerca del éxito o del fracaso escolar suelen desvincularse de los determinantes sociales y de las prácticas escolares que producen y reproducen formas de distinción, depositando así la responsabilidad en la "naturaleza individual" de cada sujeto por "su fracaso" - "la pucha yo soy el que no entiendo" es como mi culpa, es como que es mi problema"- a la vez que estructuran un veredicto, un destino. Es evidente que estos discursos no ponen en cuestionamiento el orden social ni escolar imperante como productores y reproductores de la desigualdad.

Respecto a la exclusión que se genera aun cuando se accede a la educación, resulta ilustrativo un fragmento de observación realizada en el colegio secundario, en el marco de un taller sobre los ingenios azucareros.

Se reparten textos, a cada grupo conformado, sobre la historización de la producción de caña de azúcar en la provincia de Salta, luego de unos minutos de lectura la docente pide que cada grupo comente sobre circuitos de producción y circulación del azúcar que relatan los textos. Cada grupo responde leyendo lo que marcaron en el propio texto. Finalizada la exposición de tres grupos, una estudiante levanta la mano para pedir que se hable de la producción en la Quebrada del Toro (lugar donde viven los y las estudiantes), ante lo cual la docente responde que no hay material de estudio sobre el tema, por lo que su abordaje no es posible (Observación 11-5-2018. 10:35 hs. Curso de 4to año).

En la observación se advierte claramente el abordaje de contenidos curriculares alejados de los contextos sociales propios de los estudiantes que no admiten posibilidad de diálogo ni interrelación. Una vez más, la propuesta de 
acceso real a la educación marca distancia entre los prescripto y su ejercicio en las instituciones educativas. Al respecto, Duchatzky (1996) manifiesta que "el horizonte de la inclusión no cuestiona la configuración institucional, los valores y los principios de legitimidad no se alteran, sólo se trata de acoger lo diferente desde las jerarquías ordenadoras" (p.1).

Siguiendo con los procesos contradictorios de la escuela, el relato de una estudiante universitaria indígena da cuenta de las cómo los discursos y prácticas escolares están teñidas de representaciones negativas que se asientan en el origen familiar, social y cultural de los estudiantes. La entrevistada expresa:

Cómo los docentes [del secundario] trataban a los... a los padres o a los propios estudiantes, de que bueno, no hay que enseñarles tanto porque no van a seguir estudiando o no van a hacer una carrera universitaria ni terciario" (Entrevista a ex-tutora del ProTconPO, proveniente de una comunidad indígena, p. 2 lín.5961).

La evidencia de una forma de exclusión subjetiva mediante la anticipación de que no se conseguirá el acceso a otro nivel del sistema es lo que deja ver el discurso citado. Esta anticipación anuda origen social a destino escolar y social: a tales grupos, tales destinos. Suponer que los jóvenes indígenas no podrán seguir una carrera universitaria tiene como trasfondo una concepción naturalista y esencialista del éxito o el fracaso en la escuela, supone una profundización de las diferencias como deficiencias que derivan en prácticas escolares que excluyen, segregan, anticipan.

Es como que los docentes nunca se preocuparon de que... em... que aprendamos cosas más lindas o cosas que nos puedan ayudar en... en una carrera universitaria, porque nunca pensaron que chicos de las comunidades quisieran estudiar una carrera" (Entrevista a ex-tutora del ProTconPO, proveniente de una comunidad indígena, pág. 3 lín.76-79).

Las consecuencias prácticas que tienen las representaciones y los discursos escolares derivan en "límites para enseñar" porque se supone que "no podrán aprender". De allí que la desigualdad social que afecta a los pueblos indígenas se traslada a la escuela y ésta se vuelve, una vez más, funcional a la perpetuación de un orden social.

Si bien la entrevistada accedió a la Universidad las consecuencias de no haber accedido a conocimientos esenciales para continuar en el sistema 
educativo hacen que el tránsito por el nivel universitario sea difícil, prolongado, y pueden llevar -incluso- a la deserción:

Venir a la Universidad era como que volver a empezar de nuevo, en tema de los estudios. Ahí me di cuenta que no aprendí nada en la secundaria, fue más difícil de lo que imaginaba. Hubo un momento en el que dije "hasta acá nomás llegué y me vuelvo" porque era difícil" (Entrevista a ex-tutora del ProTconPO, proveniente de una comunidad Wichí, pág. 4 lín. 132-134).

Hacer propio el discurso individualista del "fracaso" lleva a la autoexclusión, los sujetos terminan con convencerse que son la causa última de su propio fracaso en la escuela y en la sociedad. Encarnan la idea de "hasta acá llegué” como un límite de sus capacidades, de sus características intrínsecas, de su "naturaleza individual”. Kaplan (2008) señala que "cuanto más vulnerable es el estudiante que se auto-juzga, más tenderá a atribuirse el fracaso escolar a sí mismo, llegando a excluirse subjetivamente de aquello de lo que objetivamente ya está excluido” (p. 2). Esto explica cómo las desigualdades materiales objetivas se anudan con las expectativas, las esperanzas, los deseos, es decir, con lo simbólico-subjetivo, de manera que la producción y la reproducción de la desigualdad social está asegurada.

Las dinámicas de inclusión-exclusión que tienen lugar en la escuela lleva a pensar en lo que Bourdieu (1998) denomina formas negadas de eliminación. El autor alude a que el sistema educativo ya no excluye explícitamente a los estudiantes de los sectores vulnerables, su evidencia se manifiesta mediante la obligatoriedad de escolarización de jóvenes de todos los sectores sociales, no obstante, las formas de exclusión se expresan en su interior y tiene lugar sutil y paulatinamente mediante la repitencia, el abandono -por ejemplo-.

Las prácticas y discursos escolares tienen incidencia significativa en la constitución subjetiva de los y las estudiantes. Deslizar las responsabilidades hacia ellos por los "fracasos escolares" deriva en nominaciones como "inmaduros", "porros", "es lento", "no le da la cabeza", que son utilizadas a modo de descripción. Las nominaciones, en este sentido, segregan, excluyen, categorizan, establecen dualismos como exitoso/fracasado, normal/anormal, inteligente/porro. Kaplan (2008) alude las transformaciones de estas nominaciones en una suerte de marca de calidad que el sentido común social denomina como "de fábrica", producto de "la hechura" o del "sello de la cuna". La autora enfatiza en la significatividad de estos intentos de estigmatización 
social que en muchos casos son exitosos en tanto que, en general, quienes son estigmatizados asumen como propios los atributos con los cuales son clasificados, asumiendo su destino como parte de su supuesta propia naturaleza.

Las formas de nominación peyorativas y estigmatizantes cobran fuerza mayor cuando son pronunciadas por los docentes, en tanto éstos encarnan una figura autorizada y legitimada social e institucionalmente. En muchos casos los docentes, sin ser conscientes, contribuyen a reproducir el orden social en sus discursos y sus prácticas pedagógicas.

No obstante, en la escuela también se generan espacios de resistencia, contestación y sumisión en la que tienen lugar otros sentidos y significados en relación a las posibilidades y oportunidades que la escuela puede ofrecer. Así, resulta interesante cambiar la pregunta acerca de qué hacen las instituciones escolares con la población indígena, para intentar conocer "lo que hacen los pueblos indígenas con la institución escolar" (CZARNY, 2009, p. 5).

\section{La educación como posibilidad de otros horizontes}

Es innegable que al interior de las instituciones educativas las dinámicas de exclusión-inclusión conviven. Por ello, es imprescindible una mirada sociocrítica hacia su interior para develar los mecanismos ocultos que operan en la reproducción de una desigualdad social más amplia. Es necesario analizar las dinámicas de reproducción de la desigualdad escolar en relación a la matriz sociohistórica donde se fundan y cobran sentido.

Vale destacar que la escuela no es totalmente responsable de la fragmentación social, la pobreza estructural, el desempleo que atraviesa a los grupos sociales más vulnerables, entre ellos, los pueblos indígenas. Pero lo cierto es que tampoco puede ni debe permanecer ajena a las condiciones socioeconómicas y al trazo de otras posibilidades y horizontes de futuro de los y las estudiantes que asisten a ella.

En este marco, la escuela actúa consciente e inconscientemente en las dinámicas de exclusión-inclusión social, de movilización o determinismo. En contextos de desigualdad creciente, de exclusión y exterminio social, es imprescindible pensar a la escuela como espacio de justicia, democratización, movilidad, posibilidades. Los y las estudiantes que la pueblan van construyendo en ella y a partir de ella perspectivas, proyectos, formas de pensar y actuar como ciudadanos. Es en este sentido que se piensa a la escuela como espacio de 
resistencia, de cura de las heridas sociales que traen consigo los estudiantes (KAPLAN, 2008). En el marco de los discursos recuperados en la investigación se pone de relieve el valor insoslayable que se le atribuye a la escuela. A partir de ello, se busca problematizar el espacio escolar como espacio en que los mecanismos objetivos y simbólico-subjetivos entran en tensión, origen y destino se desalinean como "consecuencia" dualista. Es aquí donde entran en juego otros sentidos atribuidos a la escuela y que se considera relevante dar a conocer. Los encuestados expresan que la escuela secundaria les permitiría:

- Tener un buen trabajo en la ciudad

- Seguir con mis estudios

- Me permitiría ser alguien en la vida

- Podría saber más para ayudar a mi familia

- Me permitiría seguir estudiando para después volver a mi comunidad y ayudar

Los estudiantes depositan en la escuela, a través de su relato, esperanzas subjetivas. Estar en ella, a futuro, les permitirá algo "mejor", "conseguir un buen trabajo", "ser alguien”, "ayudar a la familia y a la comunidad". El sentido que tienen los discursos citados dista mucho de una desconfianza en la escuela.

Si bien no todos manifiestan el deseo de continuar en el sistema educativo, sí creen que la escuela les posibilitará "algo mejor". Resta problematizar sobre cuáles son los trasfondos de aquellas expresiones que aluden a que la escuela les permitirá acceder a un "buen trabajo", a "ser alguien en la vida”, puesto que dan cuenta de las concepciones que los y las estudiantes van construyendo acerca de sí mismos.

Respecto a "conseguir un buen trabajo" puede estar asociado a las condiciones materiales de existencia, es decir, a las dificultades económicas para continuar estudios superiores. Pero también puede estar relacionado a la desconfianza de sí mismo para acceder a un nivel superior. La idea de "no poder", "de que no me da la cabeza" para cursar una carrera puede estar operando como condicionante para pensar y proyectar una continuidad en el sistema educativo.

La expresión "ser alguien en la vida" interpela profundamente en tanto da cuenta de una representación de que mientras tanto, mientras no se transite y culmine la escuela no se es nadie. Parece ser que la escuela otorga identidad, da existencia, permite "ser alguien" como oposición a ser "un nadie". En las 
identidades socialmente deterioradas, la escuela juega un papel simbólico indispensable. Transforma y reivindica esa percepción acerca de sí mismo o la reafirma y perpetúa.

En lo que refiere a la escuela como posibilidad para seguir estudiando o elegir una carrera universitaria es otra de las valoraciones que evidencian los discursos. La confianza en ella para acceder a otro mundo, a otras formas de vida, de ocupación social y participación ciudadana se reflejan en los fragmentos seleccionados a continuación:

Es muy importante poder estar en la Universidad y... y ojalá que otros chicos pudieran venir y animarse a cursar (Entrevista a extutora del ProTconPO, proveniente de una comunidad indígena, pág. 4 lín.136-140).

Nunca había pensado en estudiar en la Universidad, por mi edad, pero hoy estoy súper agradecido de poder estudiar en una Universidad (Entrevista a participante del ProTconPO pág. 6 lín. 224).

Estas expresiones dan cuenta que junto a la estructura escolar y sus tendencias a la exclusión conviven expectativas de otros mundos posibles. La escuela funciona para los estudiantes indígenas a través de fuerzas centrífugas y centrípetas, simultáneamente ejerce atracción y repelencia para estos pueblos (HECHT, 2010).

En este sentido, es válido enfatizar que la escuela despliega estrategias de conservación y a su vez de transformación del orden social, por ello, se considera necesario revelar las prácticas y discursos que responden a cada una para potenciar o impulsar cambios que den lugar a otras biografías y experiencias escolares más democráticas e inclusivas.

Movilizar barreras simbólicas dentro de la escuela es batallar con prácticas y discursos eufemizados, que, bajo la apariencia de describir, prescriben. Al decir de Kaplan (2006), la escuela y sus agentes pueden derribar muros simbólicos y contrarrestar los "destinos negativos" de aquellos estudiantes atravesados por las desigualdades sociales, contribuyendo a reforzar positivamente su autoestima y sus expectativas a futuro. 


\section{CONCLUSIONES Y NUEVOS INTERROGANTES}

Ser conscientes de una tendencia académica y política que reconoce la diversidad y proclama su inclusión en el discurso pero que sin embargo está ausente en los microespacios sociales, como la escuela, lleva a pensar y a buscar cuáles son las "nuevas formas" de exclusión, bajo qué eufemismos se presentan y de qué manera se pueden develar y transformar.

El vasto abordaje legislativo que tiene Argentina respecto a los pueblos indígenas y el acceso a derechos fundamentales hace agua en su efectivización, en su concreción al interior de las instituciones por las que transitan estos grupos, pero que es innegable como avance hacia el reconocimiento.

La extensión de la educación secundaria a todos los grupos sociales es un logro y a su vez un desafío. La oportunidad educativa convive con las desigualdades sociales históricas que inciden en las estrategias pedagógicas, en las representaciones, discursos y prácticas escolares. Así pues, cuando se habla de acceso a la educación, se pone el centro sólo en el derecho a la educación, pero no se problematiza si la educación a la que se accede es adecuada, respetuosa de la diversidad cultural, la lengua, el sistema de valores de sus destinatarios.

Situarse en el dinamismo exclusión-inclusión permite comprender cómo operan los mecanismos de desigualdad, cuáles son las estrategias que pone en juego la escuela para movilizar estructuras subjetivas y posibilitar otras trayectorias escolares de los y las estudiantes indígenas. En este aspecto, resulta imprescindible problematizar el acceso como logro alcanzado, en tanto no garantiza la concreción de los distintos niveles educativos. Más que poner el foco en el punto de partida, es necesario enfocar el proceso, el tránsito por la escuela, para saber si en su interior las condiciones e intenciones de afrontamiento a la diversidad están dadas y de qué manera.

Los discursos recuperados de los y las estudiantes indígenas permiten ver las dinámicas contradictorias de exclusión-inclusión que conviven en las instituciones de educación formal, las tensiones entre lo social y lo individual y su incidencia en las subjetividades y autoestima social y escolar.

Los dualismos y las certezas quedan fuera de juego cuando se aborda a los sujetos en contextos de mutación persistente. No obstante, es posible ensayar 
formas, alternativas y posibilidades que den lugar al diálogo, al trazo de acciones que favorezcan a experiencias escolares y vitales democráticas y participativas.

El abordaje realizado en este artículo intenta dar cuenta de la complejidad del estudio, del compromiso y el distanciamiento (ELIAS, 1990) que se debe asumir en él, en tanto pensar y accionar "son fenómenos diferenciables pero inseparables" (p.51).

Finalmente, interesa dejar planteado un interrogante a modo de problematizar y reflexionar sobre el tema que se discute: ${ }_{\mathrm{C}}$ Es suficiente con mover el foco del acceso hacia la permanencia para hablar de inclusión? En estas líneas se ha cuestionado que el acceso garantice inclusión, para futuros análisis resultaría interesante plantearse si permanecer en el sistema, en las instituciones educativas, se traduce necesariamente en inclusión, si el hecho de que los estudiantes indígenas no abandonen, no sean expulsados de esas instituciones, significa que están incluidos.

\section{REFERENCIAS}

BOURDIEU, P.; SAINT MARTÍN, M. Las categorías del juicio profesoral. Actes de la Recherche en Sciences Sociales, París, v. 1, n. 3, p. 68-93, 1998.

CZARNY, Gabriela. Jóvenes indígenas y educación superior: a propósito de la discusión sobre la escolaridad como práctica para desindianizar vs. práctica para empoderar. Congreso de americanistas, 38으, 2009. Anais...

DUBET, François. La escuela de las oportunidades ¿Qué es una escuela justa? Barcelona: Editorial Gedisa, 2005.

DUSCHATZKY, Silvia. De la diversidad en la escuela a la escuela de la diversidad, 1999. Propuesta Educativa, México, v.7 n. 15, pp. 45-69.

ELIAS, Norbert. Compromiso y distanciamiento. Ensayos de sociología del conocimiento, Barcelona: Ediciones Península, 1990.

ARGENTINA. Encuentro Nacional de Organizaciones Territoriales de Pueblos Originarios (ENOTPO). Mapa Pueblos Naciones Originarias en la actualidad, 2015. 
GENTILI, Pablo. Marchas y contramarchas. El derecho a la educación y las dinámicas de exclusión incluyente en América Latina (a sesenta años de la Declaración Universal de los Derechos Humanos). Revista Iberoamericana de Educación, v. 49. pp. 19-57, 2009.

HECHT, Carolina. Todavía no se hallaron en hablar en idioma. Procesos de socialización lingüística de los niños en el barrio toba de Derqui. Múnich: Lincom Europa, 2010.

HERNÁNDEZ SAMPIERI, Roberto. Metodología de la investigación. México: MacGraw-Hill Interamricana, 2010.

ARGENTINA. Instituto Nacional de Estadísticas y Censos (INDEC) (2010) Censo Nacional de Población, Hogares y Viviendas, 2010.

KAPLAN, Carina. Talentos, dones e inteligencias. El fracaso escolar no es un destino. Buenos Aires, Argentina: Ediciones Colihue, 2008.

KAPLAN, Carina. La inclusión como posibilidad. Buenos Aires: Ministerio de Educación, Ciencia y Tecnología de la Nación, 2006.

MATO, Daniel (coord.). Educación Superior y Pueblos Indígenas en América Latina. Buenos Aires: EDUNTREF, 2015.

NARODOWSKI, María. La inclusión educativa. Reflexiones y propuestas entre las teorías, las demandas y los slogans. Revista Iberoamericana sobre Calidad, Eficacia y Cambio en Educación, v. 6, n.2, pp. 19-26, 2008.

SINISI, Liliana. Integración o Inclusión escolar: ¿un cambio de paradigma? Boletín de antropología y educación, 2010.

WIGDOROVITZ de CAMILLONI, Alicia. El concepto de inclusión educativa: definición y redefiniciones. Políticas Educativas, v. 2, n. 1, 2008.

YUNI, José y URBANO, Claudio. Técnicas para investigar: recursos metodológicos para la preparación de proyectos de investigación. Córdoba: Brujas, 2016.

Recebido em 14 de fevereiro de 2020 Aprovado em 23 de junho de 2020 